\title{
Análisis de los elementos significativos en la educación inclusiva
}

\author{
Analysis of the significant elements in inclusive education
}

* Claudia Patricia Rodríguez Andrade

** Martha Liliana Urbano Burbano

\section{Resumen}

El artículo esclarece los conceptos referentes a la educación inclusiva, visibilizando la teoría como eje esencial para la comprensión de las acciones desarrolladas en el ámbito educativo, enmarcadas en organismos y documentos nacionales einternacionales. Desde el análisis interpretativo, se retoman las percepciones que identifican las marcas enunciativas de los autores y se clasifica de acuerdo a la interrelación que se concibe de las proposiciones expuestas y la intencionalidad. De allí, se determinan los elementos y las prácticas que se deben encaminar a los integrantes de las comunidades educativas, al reconocimiento, implementación, adaptación, ajustes razonables que contribuyen a garantizar, de manera equitativa, oportunidades del aprendizaje para todos. Lo expuesto lleva a repensarse las interpretaciones y desafíos que promueven la gestión educativa como derecho fundamental.

Palabras clave: educación inclusiva, discurso, prácticas, interpretaciones, desafíos, comunidad educativa.
Rec: Febrero 2019

Acep: Julio 2019

\section{Abstract}

The article shows the concepts related to inclusive education, making the theory visible as an essential axis for the understanding of the actions developed in the educational field, framed in national and international organizations and documents. From the interpretative analysis, the perceptions that identify the enunciate marks of the authors are retaken and classified according to the interrelation that is conceived of the exposed propositions and intentionality. From there, the elements and practices are determined to show direction to the members of the educational communities to the recognition, implementation, adaptation, reasonable adjustments that contribute to guarantee equitably, learning opportunities for all. All of the above, leads to rethink the interpretations and challenges that promote educational management as a fundamental right.

Keywords: Inclusive education, speech, practices, interpretations, challenges, educational community.

\footnotetext{
* Claudia Patricia Rodríguez Andrade. Profesora e investigadora de la Fundación Universitaria Católica Lumen Gentium. Magíster en Neuropsicología y Educación de la Universidad de la Rioja. Correo electrónico cprodriguez@unicatolica.edu.co

Profesora e investigadora de la Fundación Universitaria Católica Lumen Gentium. Magíster en Educación y Diversidad de la Universidad de ** Manizales. Correo electrónico: mlurbano@unicatolica.edu.co
} 


\section{Introducción}

Actualmente, el concepto de educación inclusiva está siendo mencionado en el contexto educativo, por lo que han resultado varios interrogantes sobre qué es, quién lo respalda, cuáles son las características y elementos propios con los que se debe abordar. Por ello, se abordará desde dos instancias que dan respuesta a las cuestiones mencionadas; la primera, desde entidades y los documentos emitidos, estos son: la Declaración Universal de los Derechos Humanos DUDH (1948), que permite comprender globalmente su acción, específicamente en su artículo 26, en el cual se menciona la educación como un derecho humano básico y ha sido fundamentado en el desarrollo de acciones dignas para la vida. En este mismo sentido, la Organización de las Naciones Unidas para la Educación, la Ciencia y la Cultura UNESCO (2004), encargada de la gestión en la educación y basada en cuatro aspectos claves para su desarrollo: el primero, como un derecho básico que permitirá tener una sociedad más justa; el segundo, encaminado desde la calidad educativa; el tercero, pensado desde la creación de una educación para todos; y el último, centrado en la inclusión de grupos minoritarios. De la misma forma, la Agenda 2030 (2016) como plan mundial, específicamente para este caso en el objetivo 4, esto es: retoma la garantía de la educación inclusiva, equitativa y de calidad en pro de fomentar oportunidades de aprendizaje permanente para todos. Por otro lado, en Colombia, el Ministerio de Educación Nacional MEN (2017) establece su visión en las prácticas, políticas y culturas para minimizar las barreras de aprendizaje y participación.

Por otra parte, se retoman conceptos literales desde autores que permitirán esclarecer las percepciones que serán insumo esencial para determinar los factores que convergen de manera individual y colectiva. Inicialmente, Echeita (2017), quien ha contribuido en asesorías referentes al tema internacionalmente (Unesco, OCDE, OEI entre otras), coordinador del Consorcio en Educación; seguido, Blanco, directora de la oficina de la Organización de los Estados Americanos OIE de Chile, ha caracterizado América Latina y el Caribe en el campo educativo; Arnaiz (2003), quien ha publicado numerosos textos en el ámbito de la atención a la diversidad. Finalmente, Parra, investigador de la Universidad Sergio Arboleda de Bogotá y director del Instituto Nacional para ciegos INCI.

\section{Metodología}

Se realizó una revisión y clasificación de los documentos referenciados y se elaboró un gráfico de tiempos tomados sin secuencia, con la finalidad de evidenciar el proceso de enunciación y aporte a la educación inclusiva.

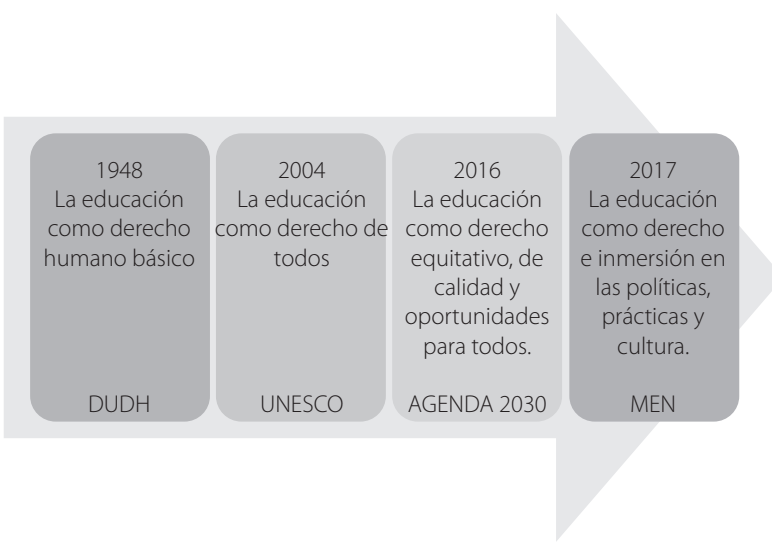

Figura 1. Enunciados que evocan a la Educación Inclusiva. Fuente: Creación propia

Maturana (1998) refiere el lenguaje como elemento trascendental en la convivencia, ya que se relaciona de manera intrínseca con las emociones, las cuales son decisivas para establecer relaciones entre los seres humanos, respondiendo a la equidad y justicia social. En este sentido, establecer el buen uso del lenguaje alrededor de las interpretaciones, encaminará a la construcción de referencias asertivas e incluyentes y evitará, por ejemplo, la emisión de mensajes que en ocasiones pueden considerarse contradictorios a la intención edu- 
cativa inclusiva. La figura anterior, como se evidencia en diversos momentos, le apuesta en los elementos expuestos insistentemente a la educación como un derecho fundamental de todos, estableciendo así, un llamado a la educación inclusiva, pensado desde la equidad y oportunidad.
Desde los autores, se retoman varios fragmentos sobre el concepto de educación inclusiva como análisis comparativo que evoca a las características y elementos a través de su proceso de enunciación demostrado en la siguiente tabla:

Tabla 1. Conceptos de Educación Inclusiva

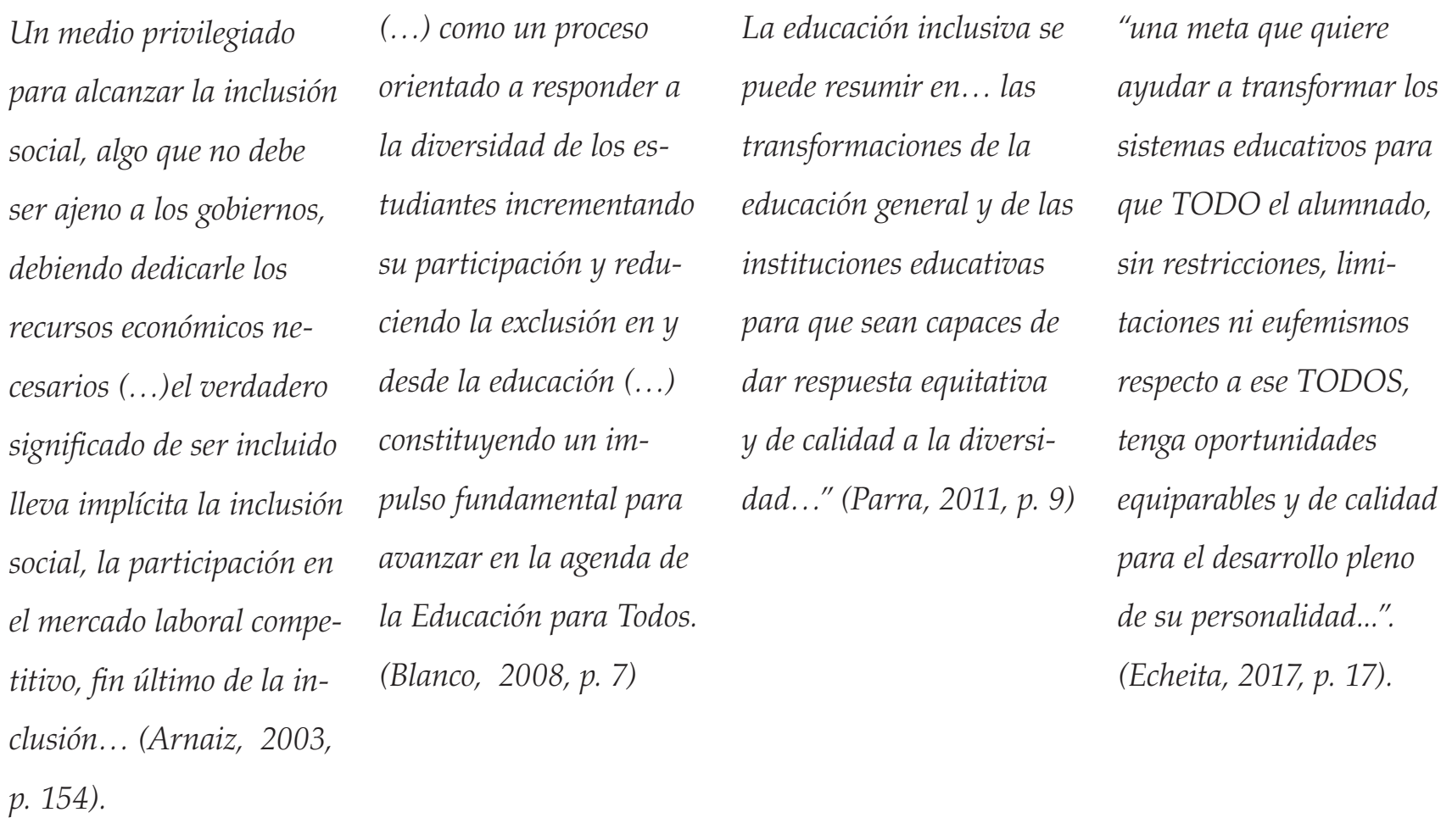

Fuente: elaboración propia. 
Es de resaltar que, a través de la enunciación individual de los autores, se encuentran algunos elementos esenciales que permiten la interrelación para conceptualizar de forma colectiva, esto es, la educación inclusiva como una meta que requiere un proceso de transformación y medio para alcanzar la participación de manera equitativa, de calidad, para todos, en camino como impulso a una inclusión social.

\section{Revisión analítica}

Retomando la postura de los autores, se evidencia para Parra (2011), la necesidad de hacer una distinción en los términos para una mejor comprensión del alcance de su significado. Así tenemos que, educación significa la construcción del conocimiento individual a partir de la incorporación e internalización de las pautas culturales; incluye el compartir conocimientos y se constituye en la base necesaria para el aprendizaje. Del mismo modo, si se analiza el término 'inclusión', desde el punto de vista educativo, es hacer efectivo para todos los derechos a la educación, contemplando la igualdad en las oportunidades, la eliminación de barreras para el aprendizaje y la participación en el contexto físico y social.

La educación inclusiva de manera articulada se enfoca en dar respuesta a tareas específicas, entre ellas: ser acogidos y compartir en lugares comunes, en los cuales intervienen emociones, acuerdos, reconocimientos que solo se aprenden estando juntos. Así mismo, garantizar la participación para ser valorados y queridos entre todos, en pro de generar bienestar y respeto por las diferencias. Seguidamente, responder al aprendizaje de forma personalizada para que los estudiantes asimilen y progresen en el desarrollo de las competencias. Finalmente, contribuir a la sostenibilidad medioambiental, como la construcción racional de sociedad y planeta, puesto que serán las que garanticen una inclusión en su totalidad (Echeita, 2017).

En el campo educativo, Arnaiz, en su texto denominado Sobre la atención a la Diversidad, aclara que algunos principios de la educación inclusiva son: 1 . Clases que acogen la diversidad, es decir, aulas donde los estudiantes puedan desarrollar una atmósfera social que les permite aprender desde varios niveles $\mathrm{y}$, al tiempo, encontrar rasgos que los identifican y los ayudan a construirse de manera eficaz y fuerte. 2 . Un currículo más amplio que fomente el desarrollo de habilidades equivalentes a sus necesidades y posibilidades. 3. Enseñanza y aprendizaje interactivo, los cuales están ligados a la pedagogía que estimulan el trabajo cooperativo, creativo e interactivo. 4. Apoyo a los profesores, encaminado a romper el aislamiento profesional, individualismo que niega las posibilidades del trabajo con otros adultos; por ende, la cooperación de otros profesores en la preparación y valoración continua, enriquece en varios aspectos. Finalmente, 5 . La participación paterna como parte del proceso significativo y de planificación que llevará a un mayor conocimiento de los estudiantes.

Desde esta perspectiva, se permite una visión más amplia de los elementos a tener en cuenta en la educación inclusiva, logrando vincular los requerimientos a nivel físico, condiciones de las aulas y sus materiales; además de la construcción estructural, en tanto filosofía, misión y visión de los procesos de enseñanza - aprendizaje, que se traducen en los documentos institucionales que guían el quehacer cotidiano de las instituciones educativas y junto a ello los procesos de acompañamiento a los docentes, quienes requieren de espacios de actualización, reflexión y planeación permanente; todo esto con el único propósito que la educación se convierta en espacio de interactividad para el desarrollo de competencias sociales, vinculando de manera más clara a los padres de familia y a la sociedad en general.

Blanco, refiere que en los países de América latina, se presenta el panorama educativo a partir de las realidades evidenciadas, por consiguiente, la ruta y recorrido hacia la educación inclusiva puede ser determinada por algunos desafíos como: mayor cobertura y calidad en la primera infancia; progresión oportuna y conclusión de la primaria y secundaria; eliminación de formas de discriminación; mejoramiento en los 
procesos educativos para el acceso y la apropiación del conocimiento; inversión en la formación y condiciones adecuadas para los docentes; desarrollo de sistemas de apoyo en atención a la diversidad; fortalecimiento de sistemas integrales de protección y promoción social; acceso al uso de las TIC; aumento de mayor inversión y hacer más equitativo el gasto público en educación y adelanto en sistemas de información desagregada por factores de inclusión (2014). Por lo tanto, todo está acorde a las necesidades del contexto y esto es, en principio, la conformación de equipos que identifiquen las necesidades para dar inicio, no precisamente desde lo más difícil, más vale de aquellos pequeños elementos considerados que pueden ir modificando.

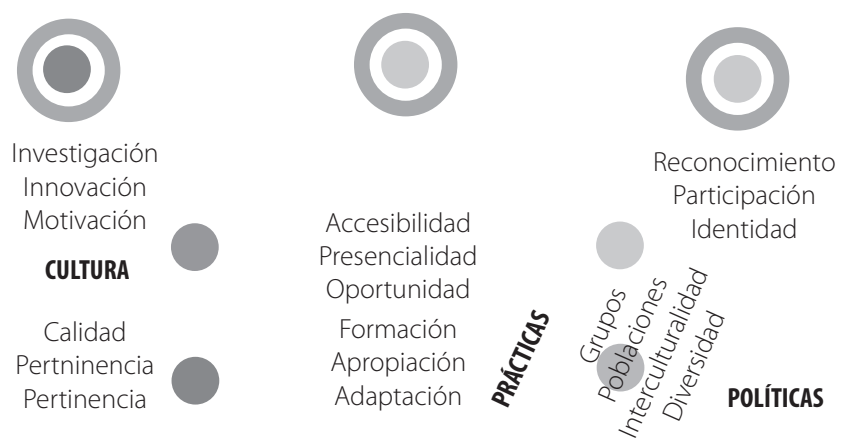

Figura 2. Elementos de la Educación Inclusiva Fuente: Elaboración propia

Tabla 2. Conceptos de la práctica educativa
Cada uno de los focos (políticas, prácticas y cultura) se retoman de acuerdo a la visión que el Ministerio de Educación Nacional le apuesta, de allí se construyen grupos de palabras asociadas a una misma intencionalidad que aportan significativamente a una educación para todos y dejan entrever que, aunque las posturas parezcan ser independientes, cada uno aporta al compromiso que se desarrolla en la educación desde la sinergia de sus postulados.

Por ello, desde este análisis también se retomaron características discursivas sobre las prácticas educativas que permiten aportar a las acciones continuas a desarrollar en los contextos educativos, con el objetivo de aportar y garantizar el fortalecimiento de los factores que deben infundir en la educación inclusiva.

Como primordial aspecto, se debe considerar que todo sujeto, de manera cotidiana, se desenvuelve en diversas situaciones y espacios, los cuales lo llevan a estar en constantes prácticas - acciones, que implican el desarrollo de procesos que vinculan sus conocimientos y experiencias previas, situaciones que están determinadas por el campo sociocultural donde se encuentra inmerso el sujeto: desde esta perspectiva, se presenta el siguiente análisis sobre práctica educativa:
"(...) las prácticas educativas se constituyen de distintas estrategias discursivas y semióticas que comprometen tanto a alumnos como docentes en la construcción de supuestos relacionados con determinados conocimientos y formas de actuación" (Chávez Jaramillo, 2014, p.166).
La práctica se convierte en una unidad de análisis, desde la cual se puede plantear un estudio de la evolución educativa, de alli Kemmis (1999), propone cuatro planos,

"(...) las intenciones del profesional (...) en el plano social (...)plano histórico (...) se construyen en un plano político (p. 23).
"La buena práctica inclusiva debe entenderse como una actuación "situada", que adquiere sentido $y$ es viable a partir de una realidad concreta, de unos condicionantes estructurales que la hacen única e irrepetible. No hay buenas prácticas ideales, sino que dependen del contexto en el que se desarrollan" (OEI, 2009).
"La mejora con una orientación inclusiva ocurre cuando los adultos y los estudiantes vinculan sus acciones a valores inclusivos y desarrollan en conjunto prácticas educativas que convergen hacia esos valores (...) estas acciones serán (...) plan formal de mejora que refleje tales valores inclusivos" (Booth Ainscow 2015, p. 18). 
De forma colectiva, se puede considerar que las prácticas educativas están ligadas a las acciones, estrategias, estructuras, valores de los miembros de las comunidades educativas y se construyen mediante la interacción de los diferentes grupos de sujetos que participan de la consolidación del proceso educativo; entendidos como aquellos que aportan los conocimientos que se incluyen en él, asumiéndolos como relevantes y esenciales para el plano social.

Chávez y Jaramillo (2014) aclaran que quienes conforman estos grupos están en constante cambio o evolución, en cuanto a las posibilidades de crear estrategias que ayuden a comprender el conocimiento o la información que madurará los procesos cognitivos, sociales, emocionales de cada uno de los individuos; al igual que en el grupo receptores, quienes se transforman y asumen nuevos procesos, en la mayoría de los casos, determinados por el desarrollo de nuevas tecnologías y percepciones sociales y culturales.

Para Kemmis (1993), estos planos dejan entre ver que las prácticas educativas, más que acciones explícitas, contienen elementos en su accionar que corresponden a una dimensión estructural de contenido ideológico, dentro del marco histórico y político; donde se pueden identificar estructuras de poder, en tanto son determinantes del quehacer cotidiano en el aula de clase, interviniendo de forma afirmativa las dimensiones de enseñanza-aprendizaje. Los marcos referenciales y normativos que corresponden al espacio macro, son apuestas a nivel político que en la mayoría de los casos se encuentran en debate social, se comportan desde políticas públicas cíclicas, que dan respuestas a coyunturas sociales sin modificar su trasfondo, de allí la imposibilidad de identificar revoluciones educativas significativas. Otros elementos cercanos en tanto perceptibles de cambio próximo, son los correspondientes a la construcción intencional, la cual, en la mayoría de los casos, es objeto de procesos de afirmación, contracción o modificación del plano social circundante; el cual no se escapa a las propias dinámicas dominantes.

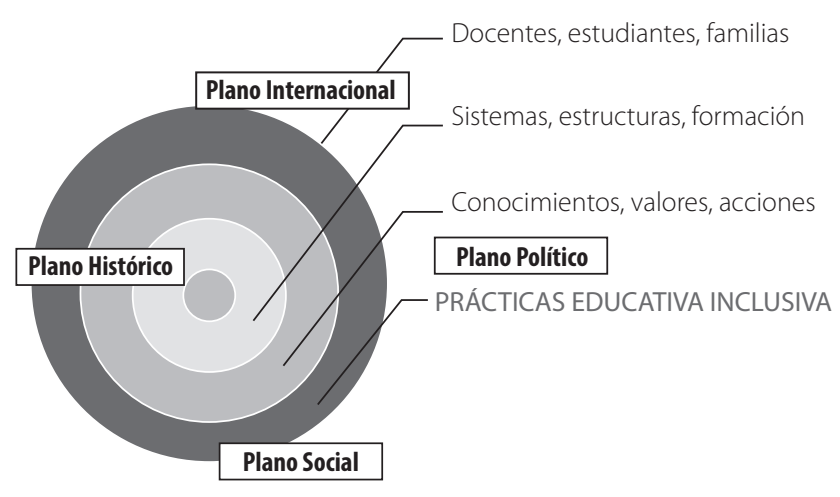

Figura 3. Elementos para la comprensión de la práctica educativa inclusiva. Fuente: Elaboración propia

De esta manera, las prácticas educativas inclusivas no deben ser de uso exclusivo de los docentes, en cuanto se reconocen otros aspectos dentro de la sociedad que exige una vinculación más estrecha de la comunidad a los procesos de inclusión; es decir, se debe ir más allá de la sensibilización, reconocimiento de la diferencia, puesto que implica un proceso permanente a todos los actores sociales que permiten una reflexión clara de lo que significa la práctica educativa inclusiva. Esto favorece el ejercicio dentro de la escuela, en tanto ofrece un escenario accesible a todos los miembros desde diferentes frentes, no solo en el escolar, sino en la adquisición de conocimientos académicos y los que corresponden al mundo de la vida, agregando la visión social de aquellos que no se ven inmersos de manera directa pero que, desde la experiencia del ejercicio, se preparan para un mundo donde se presenta la diferencia que exige el reconocimiento y acción como facilitadores en la educación.

\section{Conclusiones}

El lenguaje y la necesidad de aclarar los términos como la educación presenta el derecho que tienen los sujetos para acceder al conocimiento y desarrollar capacidades que le permitan establecer relaciones en los diferentes ámbitos en los que se moviliza, de allí su estructura clara, organizada 
que se permea especialmente por lo social, cultural y político de un país.

En el marco legal existe, desde hace muchos años, la creación de documentos que encaminan la educación como un derecho fundamental de todos; de manera insistente promueve, intrínsecamente, la inclusión, en especial de aquellos más vulnerables y que de alguna manera no pueden acceder a los contextos educativos. Por ello, es muy importante constatar desde un marco legal global y nacional, la forma cómo se asumen con el fin de verificar la interpretación que se da en el contexto al que corresponda.

El concepto de la Educación Inclusiva para las comunidades educativas, y en especial para los miembros quienes intervienen en ellas por fundamentación legislativa o teórica, convoca a brindar atención de calidad a los niños, niñas y jóvenes quienes acuden a los centros de formación de quienes no han ingresado. De allí, es necesario repensar sobre las acciones que movilizan la defensa de los derechos humanos y aportan a la dinámica social que permite vincular al sujeto y potencializar los diferentes medios para este fin.

La inclusión, desde un marco general, permite reconocer los escenarios a los que se enfrentan los sujetos (económico, social, cultural, políticos), los cuales evocan un enfoque para todos como posibilidad de reconocimiento a la diversidad y condición inherente a los seres humanos, encauzada, como diría Fernández (2003), en las razones sociales y morales que implican una educación de calidad, capaz de contribuir al buen sentido social, basado desde la autonomía en el diseño escolar de cada país y pensado para adaptarse a las necesidades de los niños, niñas y jóvenes en su realidad.

Reconocer los elementos que convergen en la educación inclusiva, fortalece los procesos participativos, generando la visibilización de cualidades y riquezas sociales presentes en el contexto; del mismo modo, permite avanzar en el reconocimiento de la diversidad en un espacio ideal que solo es posible dentro de ambientes equitativos, justos y de calidad, con acceso a medios eficaces para la apropiación de conocimiento, incluyendo las TIC. De lo mencionado, se considera que la ruta hacia la educación inclusiva requiere del compromiso social y soporte de los agentes que conforman y gestionan las instituciones educativas.

Las prácticas enmarcadas desde los cuatro planos (intencional, político, histórico, social) y con el entramado conceptual y constructivo, determina los procesos de selección, desde las cuales se visualizan diferentes agentes, momentos formativos, métodos y recursos, que evidencian la intencionalidad primaria de contribuir en la adquisición de conocimientos desde la consolidación de habilidades propias del aprendizaje en la diversidad.

\section{Recomendaciones}

La Educación Inclusiva, menciona la necesidad de hacer una distinción en los términos para una mejor comprensión del alcance de su significado. Así tenemos que, educación significa la construcción del conocimiento individual, a partir de la incorporación e internalización de las pautas culturales que incluye el compartir conocimientos y se constituye como base necesaria para el aprendizaje.

La eliminación de barreras para el aprendizaje y la participación los escenarios físico y social debe ser una tarea constante en todos los contextos educativos, independientemente de las situaciones que se presenten, en especial de aquellas que están permeadas por el sistema estándar homogéneo que desfigura el reconocimiento a la atención de los estudiantes en la diversidad.

La creación de nuevos escenarios en la educación convoca a estar en constante investigación, formación y revisión de las acciones, partiendo de las condiciones sociales y los objetivos planteados dentro del sistema, puesto que serán, en cierta medida, los que se conviertan en valores de una educación con prácticas educativas inclusivas. 


\section{Referencias bibliográficas}

Arnaiz, S. (2003) Educación Inclusiva: Una escuela para todos. Ciudad: Ediciones Aljibe. Recuperado de http://webdocente.altascapacidades.es/Educacion\%20inclusiva/474-texto_completo_1_atencion_a_la_diversidad.pdf

Ainscow, M y Miles, S. (2008). Por una educación para todos que sea inclusiva. Revista Perspectivas, XXXVIII, intervalo de páginas.

Bernal G. A (1998) Educar en y para la diversidad. Comunidad Educativa. Ciudad: Murcia España.

Blanco, R (2005). Orientaciones para la inclusión. Asegurar el acceso a la Educación para todos. París: UNESCO.

Blanco, R (2014). Avances y Desafíos de la Educación inclusiva en Iberoamérica. Inclusión Educativa en América Latina: caminos y recorridos por recorrer. Metas Educativas 2021. Organización de los Estados Iberoamericanos para la Educación la ciencia y la cultura. Madrid: España (Pp. 11-35)

Booth T y Ainscow M. 2015. Guía para la Educación Inclusiva: Desarrollando el aprendizaje y la participación en los centros escolares. Adaptación de la $3^{\circ}$ Edición revisada del Index for inclusion.País

CEPAL. (2018). Agenda 2030 y los Objetivos de Desarrollo Sostenible, Una oportunidad para América Latina y el Caribe. Publicación de las Naciones Unidas. Santiago de Chile. Recuperada el 1 de octubre de 2018. Recuperado de https://repositorio.cepal.org/bitstream/handle/11362/40155/10/S1700334_es.pdf

Declaración de los Derechos Humanos. (1948). Adoptada y proclamada por la Asamblea General en su resolución 217 A (III), de 10 de diciembre de 1948. Recuperado de https://www.ohchr.org/EN/UDHR/Documents/ UDHR_Translations/spn.pdf
Echeita S. G. (2017). Educación inclusiva. Sonrisa y lágrimas. Universidad de Oviedo. Aula Abierta 46, págs.1724. Recuperado de https://dialnet.unirioja.es/servlet/ articulo?codigo $=6060634$

Fernández, A. (2003). Educación Inclusiva: Enseñar y Aprender entre la Diversidad. Revista Digital Umbral 2000, 13. Recuperado de http://www.reduc.cl/wp-content/uploads/2014/08/EDUCACI\%C3\%93N-INCLUSIVA.pdf

Maturana, H (1998). Emociones y lenguaje en Educación y política. Belo Horizonte: UFMG.

Kemmis, S. (1993). El Curriculum: Más allá de la teoría de la reproducción. Madrid: Morata

Organización de Estados Iberoamericanos para la Educación, la Ciencia y la Cultura OEI (2009). Guía para para reflexión y valoración de las prácticas inclusivas.. Madrid: Editorial.

Parra,. C. (2011). Educación inclusiva: un modelo de diversidad humana. Revista educativa y desarrollo social. Ciudad: Editorial.

Maturana, H (1998). Emociones y lenguaje en Educación y política. Belo Horizonte: UFMG.

UNESCO (2004) Temario abierto sobre la Educación Inclusiva. Oficina Regional de Educación de la UNESCO para América Latina y el Caribe OREALC / UNESCO. Recuperado de http://unesdoc.unesco.org/images/0012/001252/ 125237so.pdf

UNESCO (2016). Educación 2030. Declaración de Incheon y Marco de Acción para la realización del objetivo de Desarrollo Sostenible 4. Hacia una educación inclusiva, equitativa y de calidad y un aprendizaje a lo largo de la vida para todos. Recuperado de http://unesdoc.unesco.org/images/0024/002456/245656s.pdf 